\title{
Oral Contraceptives for Endometriosis Associated Pain: A Randomized Controlled Trial
}

\author{
Swailum MB*, Wahba KA, Labib KM and Islam \\ BA \\ Department of Obstetrics and Gynecology, Faculty of \\ Medicine-Ain-Shams University, Cairo, Egypt \\ *Corresponding author: Mohamed Abdelhafez \\ Swailum, Department of Obstetrics and Gynecology, \\ Faculty of Medicine - Ain-Shams University, Cairo, Egypt \\ Received: November 05, 2017; Accepted: November \\ 30, 2017; Published: December 07, 2017
}

\author{
Abstract \\ Objective: To determine the efficacy and safety of oral contraceptive \\ preparation in the treatment of painful symptoms associated with the diagnosis \\ of endometriosis.
}

Design: Randomized controlled clinical trial- single blinded technique.

Settings: Clinical trial sited in Egypt.

Patient: Seventy patients with painful symptoms associated with endometriosis.

Intervention: Patients were randomly assigned to receive OCPs + NSAID's or Tri-B tablets + NSAID's as a placebo. Participants allowed to use pain medications as needed during the trial.

Main Outcome Measure: After four cyclic treatments, Pain was assessed using numbering rating scale (Wong-Baker FACES $₫$ Pain Rating Scale Figure 1) from (0 to 10) for each of the three symptoms: dysmenorrhea, dyspareunia and non-menstrual pain, also recurrence and frequency of pain and use of pain killers after treatment were measured.

Result: Dysmenorrhea pain was significantly reduced after treatment for patient group (the pain score reduced to $2.69 \pm 1.95$, while in the placebo group dysmenorrhea pain score was $4.66 \pm 2.17$. As regard dyspareunia pain was reduced after treatment (the pain score reduced to $1.74 \pm 1.65$, while for placebo group there are slight reduction $2.29 \pm 2.01$ ), Non-menstrual pain was reduced after treatment for patient group $(1.89 \pm 2.05)$, while for placebo group pain score was $(2.14 \pm 2.25)$, the recurrence and frequency of pain and the use of pain killer was lower in patient group than placebo group.

Conclusion: The present study demonstrated that continues use of low dose OCPs are an effective treatment for pain associated with endometriosis with few adverse effects in women who did not wish to get pregnant in the near future.

Keywords: Oral Contraceptive (OC); Endometriosis; Dysmenorrheal; Dyspareunia; Non-menstrual pelvic pain

\section{Introduction}

Endometriosis, the presence of endometrial glands and stroma outside of the endometrial cavity, represents one of the most challenging gynecologic conditions to manage given its insidious onset, surgical diagnosis, association with pelvic pain and infertility, and often progressive nature. Endometriosis is a chronic disease affecting at least $10 \%$ of reproductive-aged women, but is found in approximately $40 \%$ of infertile women and up to $90 \%$ of women with pelvic pain.

The classic triad of endometriosis symptoms, dysmenorrhea, dyspareunia, and dyschesia, raises clinical suspicion for this disorder [1].

Medical and surgical treatments are mainstays in the management of endometriosis, and different approaches are dictated by the pleiotropic manifestations of the disease as well as underlying patient characteristics. In general, medical treatment options are limited when fertility is desired because of the ovarian suppression inherent in their mechanisms of action.

The medical treatment options for endometriosis rely on the suppression of endometriosis by manipulating the hormonal milieu because endometriosis growth and activation are stimulated by estrogen, and both estrogen and progesterone receptors are present in ectopic endometrial tissue.

Hormonal contraceptives containing both Ethinyl Estradiol (EE) and progestin can be used in a cyclic or continuous fashion for the treatment of endometriosis. Continuous use appears to result in better pain control [2].

\section{Materials and Methods}

The study patients were recruited from women attending outpatient clinic at (Ain-Shams University Maternity Hospital). Seventy women of reproductive age who complained of symptoms 
referred to the diagnosis of endometriosis were randomly assigned as patient and placebo group those will receive (OCPs + NSAID's) and (Tri-B tablets +NSAID's) as a placebo in continues pattern for four successive cycles, respectively.

The study patients must have had moderate or severe dysmenorrhea, scoring higher than three points at the admission on verbal rating scale. Each patient underwent a pre-recruitment evaluation, consisting of a general medical and gynecologic history, physical and pelvic examination, clinical evaluation of signs and symptoms, patient evaluation of pain, and review of the menstrual record, pain scoring dysmenorrhea, dyspareunia and non menstrual pain and recording details about the use of analgesics.

Inclusion criteria: Age between 20-42 years old, Patient diagnosed as endometriosis based on laparoscopic or surgical diagnosis or presence of chocolate cysts and Patient must have one painful symptom associated with endometriosis.

\section{Exclusion criteria}

Patients who had received any hormonal therapy within the last three months patients, Patients with contraindications to the OCP, Patients had surgery other than biopsy had been performed in the three months prior to recruitment, Urinary tract infection causing pelvic pain, local focal uterine lesion or ovarian cyst causing pain and GIT disease causing dyschazia or pelvic pain eg. GI ulcer, piles, ulcerative colitis, etc.

Primary outcome measures were improvement of pain symptoms of endometriosis: dysmenorrhea, dyspareunia non-menstrual pain and improvement of any other pain symptom of endometriosis such as (Post-coital pain, dyschezia and/or cyclical pain not associated with menstruation), also measurement of any side effects occurring during therapy (including pregnancy).

After four month of therapy, Pain was reassessed at the end of treatment for each of the three symptoms. Also recurrence, frequency of pain and the use of pain killers were reassessed at end of treatment. The numbers of women at trial entry and at the end of treatment who had either no pain or any pain (mild, moderate or severe) were calculated from the data presented.

\section{Statistical analysis}

Data were analyzed using Statistical Program for Social Science (SPSS) version 20.0. Quantitative data were expressed as mean \pm Standard Deviation (SD). Qualitative data were expressed as frequency and percentage.

-P-value $<0.05$ was considered significant.

-P-value $<0.001$ was considered as highly significant.

-P-value $>0.05$ was considered insignificant.

\section{Results}

Demographic characteristics and disposition of patient group and placebo group are demonstrated in Table 1. This table shows that the demographic characteristics of the two groups were similar. The mean age of the patient and placebo groups was $~ 31$ years (range $23-42$ ), and $\sim 30$ years (range $22-42$ ), respectively. The mean parity of both groups was $\sim 1$ (range $0-3$ ), and BMI mean was 25.5 and 25.6 for patient group and placebo group respectively. None of them

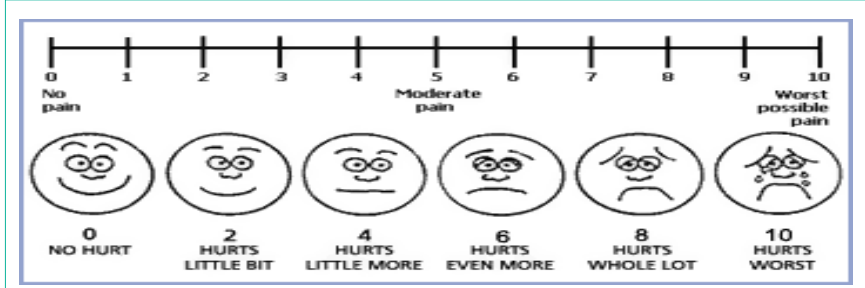

Figure 1: Wong-Baker FACES ${ }^{\circledR}$ Pain Rating Scale.

Table 1: Comparison between groups according anthropometric measurements. \begin{tabular}{|l|l|l|l|l|}
\hline Demographic measurements & Patients & Control & t-test $/ z^{*}$ & p-value
\end{tabular}

\begin{tabular}{|c|c|c|c|c|}
\hline 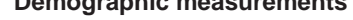 & & & & 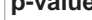 \\
\hline \multicolumn{5}{|l|}{ Age (years) } \\
\hline Mean $\pm S D$ & $31.11 \pm 5.47$ & $30.49 \pm 6.17$ & 0.204 & 0.653 \\
\hline Range & $23-42$ & $22-42$ & & \\
\hline \multicolumn{5}{|l|}{ Parity } \\
\hline Median(IQR) & $1(2)$ & $1(2)$ & 0.168 & 0.684 \\
\hline Range & $0-3$ & $0-3$ & & \\
\hline \multicolumn{5}{|l|}{ BMI } \\
\hline Mean $\pm S D$ & $25.49 \pm 3.18$ & $25.62 \pm 3.21$ & 0.029 & 0.865 \\
\hline Range & 19.38-32.03 & 19.38-32.03 & & \\
\hline
\end{tabular}

As regard table one, this table shows no statistically significant difference between groups according anthropometric measurements.

Table 2: Comparison between groups according to dysmenorrhea post treatment

\begin{tabular}{|c|c|c|c|c|}
\hline Dysmenorrhea & Patients & Control & t-test & p-value \\
\hline Mean $\pm S D$ & $2.69 \pm 1.95$ & $4.66 \pm 2.17$ & \multirow{2}{*}{15.98} & \multirow{2}{*}{$<0.001$} \\
\hline Range & $0-7$ & 9-Feb & & \\
\hline \multicolumn{5}{|l|}{ Recurrence } \\
\hline No & $29(82.9 \%)$ & $14(40.0 \%)$ & \multirow{2}{*}{13.57} & \multirow{2}{*}{$<0.001$} \\
\hline Yes & $6(17.1 \%)$ & $21(60.0 \%)$ & & \\
\hline \multicolumn{5}{|l|}{ Frequency } \\
\hline Mild & $3(50 \%)$ & $8(38.1 \%)$ & \multirow{3}{*}{13} & \multirow{3}{*}{0.005} \\
\hline Moderate & $3(50 \%)$ & $8(38.1 \%)$ & & \\
\hline Severe & $0(0.0 \%)$ & $5(23.8 \%)$ & & \\
\hline \multicolumn{5}{|l|}{ Use of pain killers } \\
\hline No pain & $1(25 \%)$ & $6(28.6 \%)$ & \multirow{4}{*}{9.611} & \multirow{4}{*}{0.022} \\
\hline Mild & $4(50 \%)$ & $5(23.8 \%)$ & & \\
\hline Moderate & $1(25 \%)$ & $7(33.3 \%)$ & & \\
\hline Severe & $0(0 \%)$ & $3(14.3 \%)$ & & \\
\hline
\end{tabular}

As regard table two, this table shows highly statistically significant difference between groups according to dysmenorrhea.

had hypertension or recently used any hormonal treatment. There is no statistically significant difference between groups according to demographic characteristics and anthropometric measurements

Endometriosis associated Pain was assessed at start of study in Numerical Rating Scale (NRS) for each type of pain pretreatment in both groups, each pain scale had score from 0 to 10 resembling no pain to worst possible pain. This date was shown in Table 2, 3 .

Dysmenorrhea means score was $5.80 \pm 1.68$ (range 4-10) for patient group and $5.60 \pm 1.52$ (range 4-10) for control group. Dyspareunia mean score was $2.57 \pm 1.75$ (range $1-8$ ) and $2.37 \pm 1.99$ (range $0-7$ ) for 
Table 3: Comparison between groups according to dyspareunia post treatment. This table shows no statistically significant difference between groups according to dyspareunia.

\begin{tabular}{|c|c|c|c|c|}
\hline Dyspareunia & Patients & Control & t-test & p-value \\
\hline Mean $\pm S D$ & $1.74 \pm 1.65$ & $2.29 \pm 2.01$ & \multirow{2}{*}{1.526} & \multirow{2}{*}{0.221} \\
\hline Range & $0-7$ & $0-7$ & & \\
\hline \multicolumn{5}{|l|}{ Recurrence } \\
\hline No & $32(91.4 \%)$ & $29(82.9 \%)$ & \multirow{2}{*}{1.148} & \multirow{2}{*}{0.284} \\
\hline Yes & $3(8.6 \%)$ & $6(17.1 \%)$ & & \\
\hline \multicolumn{5}{|l|}{ Frequency } \\
\hline Mild & $1(33.3 \%)$ & $1(16.7 \%)$ & \multirow{2}{*}{1.433} & \multirow{2}{*}{0.488} \\
\hline Moderate & $2(66.7 \%)$ & $5(83.3 \%)$ & & \\
\hline \multicolumn{5}{|c|}{ Use of pain killers } \\
\hline No pain & $0(0.0 \%)$ & $1(25 \%)$ & \multirow{3}{*}{5.065} & \multirow{3}{*}{0.079} \\
\hline Mild & $3(100 \%)$ & $1(25 \%)$ & & \\
\hline Moderate & $0(0.0 \%)$ & $4(50 \%)$ & & \\
\hline
\end{tabular}

Table 4: Comparison between groups according to non-menstrual pain post treatment.

\begin{tabular}{|c|c|c|c|c|}
\hline Non-menstrual pain & Patients & Control & t-test & p-value \\
\hline Mean $\pm S D$ & $1.89 \pm 2.05$ & $2.14 \pm 2.25$ & \multirow{2}{*}{0.249} & \multirow{2}{*}{0.619} \\
\hline Range & $0-8$ & $0-9$ & & \\
\hline \multicolumn{5}{|l|}{ Recurrence } \\
\hline No & $30(85.7 \%)$ & $28(80.0 \%)$ & 0.402 & 0.526 \\
\hline Yes & $5(14.3 \%)$ & $7(20.0 \%)$ & & \\
\hline \multicolumn{5}{|l|}{ Frequency } \\
\hline Mild & $4(80 \%)$ & $4(57.1 \%)$ & & \\
\hline Moderate & $1(20 \%)$ & $1(14.3 \%)$ & & \\
\hline Severe & $0(0 \%)$ & $2(28.6 \%)$ & & \\
\hline \multicolumn{5}{|l|}{ Use of pain killers } \\
\hline No pain & $4(80 \%)$ & $2(28.6 \%)$ & \multirow{4}{*}{3.583} & \multirow{4}{*}{0.31} \\
\hline Mild & $1(20 \%)$ & $2(28.6 \%)$ & & \\
\hline Moderate & $0(0 \%)$ & $1(14.3 \%)$ & & \\
\hline Severe & $0(0 \%)$ & $2(28.6 \%)$ & & \\
\hline
\end{tabular}

As regard table four, this table shows no statistically significant difference between groups according to non-menstrual pain.

patient and control group respectively and non-menstrual pain mean score was $2.71 \pm 2.36$ (range $0-9$ ) for patient group and $2.29 \pm 2.36$ (range 0-9) for control group.

There is no statistically significant difference between groups according to (NRS).

At the end of treatment, pain was reassessed for each of the three symptoms. Dysmenorrhea pain was significantly reduced after treatment for patient group (the pain score reduced to $2.69 \pm 1.95$ (range 0-7), while in the placebo group dysmenorrhea pain score was $4.66 \pm 2.17$ (range 2-9).

The recurrence of pain was higher in placebo group than in patient group ( 21 vs. 6 patients) with higher frequency of pain in placebo group ( 3 patients had mild pain and 3 patients had moderate pain in patient group while 8 patients had mild pain, 8 patients had
Table 5: Difference between pre and post according to NRS in patients and control group.

\begin{tabular}{|c|c|c|c|c|c|}
\hline \multicolumn{6}{|c|}{ Patient group } \\
\hline \multirow{2}{*}{ NRS } & \multirow{2}{*}{\begin{tabular}{|c} 
Pre \\
Mean $\pm S D$
\end{tabular}} & \multirow{2}{*}{\begin{tabular}{|c|} 
Post \\
Mean \pm SD
\end{tabular}} & \multirow{2}{*}{ Mean Diff. } & \multicolumn{2}{|c|}{ Paired Sample t-test } \\
\hline & & & & $\mathbf{t}$ & p-value \\
\hline Dysmenorrhea & $5.80 \pm 1.68$ & $2.69 \pm 1.95$ & -3.11 & 19.765 & $<0.001$ \\
\hline Dyspareunia & $2.57 \pm 1.75$ & $1.74 \pm 1.65$ & -0.83 & 2.938 & 0.039 \\
\hline Non-menstrual pain & $2.71 \pm 2.36$ & $1.89 \pm 2.05$ & -0.82 & 2.465 & 0.041 \\
\hline \multicolumn{6}{|c|}{ Control group } \\
\hline \multirow{2}{*}{ NRS } & Pre & Post & \multirow{2}{*}{ Mean Diff. } & \multicolumn{2}{|c|}{ Paired Sample t-test } \\
\hline & Mean \pm SD & Mean \pm SD & & $\mathbf{t}$ & p-value \\
\hline Dysmenorrhea & $5.60 \pm 1.52$ & $4.66 \pm 2.17$ & 0.94 & 4.91 & 0.022 \\
\hline Dyspareunia & $2.37 \pm 1.99$ & $2.29 \pm 2.01$ & 0.09 & 0.902 & 0.373 \\
\hline Non-menstrual pain & $2.29 \pm 2.36$ & $2.14 \pm 2.25$ & 0.14 & 0.334 & 0.741 \\
\hline
\end{tabular}

moderate pain and 5patients had severe pain in control group).

Also the use of pain killer was much higher in placebo group (15 vs. 10 in placebo group and patient group respectively). There is highly statistically significant difference between groups $(\mathrm{P}$. value $<0.001)$.

As regard dyspareunia pain was reduced after treatment (the pain score reduced to $1.74 \pm 1.65$ (range 0-7)), while for placebo group there are slight reduction $(2.29 \pm 2.01)$ (range 0-7).

The recurrence of pain was low in both groups but higher in placebo group than in patient group ( $3 \& 6$ patients in OCP and placebo group respectively). Also the use of pain killer was low in both groups. The frequency of pain in placebo group was also higher ( 1 patient had mild pain and 2 patients had moderate pain in patient group while 1 patient had mild pain, 5 patients had moderate pain in control group).

Also the use of pain killer was higher in placebo group ( $3 v s .5$ in patient group and placebo group respectively). The data shows no statistically significant difference between groups according to dyspareunia post-treatment $(\mathrm{P}$. value $=0.221)$.

Non-menstrual pain was reduced after treatment for patient group (1.89 \pm 2.05$)$ (range 0-8), while for placebo group pain score was $(2.14 \pm 2.25)$ (range $0-8)$. The recurrence of pain was low in both groups (5 and 7 patients in OCP and placebo group respectively).

As regard frequency of pain; 4 patients had mild pain and 1 patient had moderate pain in patient group while 4 patients had mild pain, 1 patient had moderate pain and 2 patients had severe pain in control group). The use of pain killer was higher in placebo group ( $1 v s .5$ in patient group and placebo group respectively) (Table 4,5 ).

The data shows no statistically significant difference between groups according to non-menstrual pain post treatment.

\section{Discussion}

Endometriosis is a common gynecological condition which affects many women of reproductive age worldwide and is a major cause of pain and infertility. The symptoms of endometriosis include dysmenorrhea, dyspareunia, and dyschezia, chronic pelvic pain, irregular uterine bleeding and/or infertility. Endometriosis represents 
one of the most

Challenging gynecologic conditions to manage, given its insidious onset, surgical diagnosis, association with pelvic pain and infertility raises clinical suspicion for this disorder. However, the substantial overlap of endometriosis symptoms with other conditions causing pelvic pain, gynecologic and non-gynecologic, combined with the limitation of pelvic examination in detecting endometriosis, makes clinical diagnosis challenging.

Furthermore, the amount of endometriosis present does not necessarily correlate with symptoms, and therefore, the usefulness of available staging systems is limited.

Many studies have been established in the past few years to determine the efficacy of oral contraceptive in treatment of endometriosis associated pain for example Harada and colleagues who Evaluate the efficacy of a low-dose oral contraceptive pill for dysmenorrhea associated with endometriosis, a Placebo-controlled, double-blind, randomized trial held in 2008 and was sited in Japan over one hundred patients. After four cycles Total dysmenorrhea scores assessed by the verbal rating scale were significantly decreased at the end of treatment in both groups dysmenorrhea in the OCP group was significantly milder than in the placebo group [3]. Another study by Harada and colleagues in 2011, which evaluate the efficacy of a low-dose oral contraceptive pills for primary dysmenorrhea, a Placebo-controlled, double-blind, randomized trial sited in Japan over one hundred fifteen patients for four cycles and found that reduction in total dysmenorrhea score and verbal rating scale and Visual Analog Scale (VAS) before and after treatment was significantly higher in the OCP group than in the placebo group, no serious adverse events occurred [4].

Also a systemic review by Cochrane Collaboration was done in 2009to assess the effects of the Oral Contraceptive Pill (OCP) in comparison to other treatments for painful symptoms of endometriosis in women of reproductive age, and found that only one study met the inclusion criteria, in which a total of 57 women were allocated to two groups to compare an OCP to a GnRH analogue over treatment period of six months, the result shows no evidence of a difference in outcomes between the Oral Contraceptive Pill (OCP) studied and GnRH analogue was as effective as a $\mathrm{GnRH}$ analogue in treating for endometriosis-associated painful symptoms of endometriosis [5].

We carried out an RCT to evaluate the efficacy of continues use of low dose OCPs for the treatment of different types of pain associated with endometriosis. Study was held from February 2016 to December, the study patients were recruited from women attending outpatient clinic at (Ain-Shams University Maternity Hospital). Seventy women of reproductive age who complained of symptoms referred to the diagnosis of endometriosis were randomly assigned as patient and placebo group those will receive (OCPs + NSAID's) and (Tri-B tablets +NSAID's) as a placebo in continues pattern for four successive cycles, respectively.

After four month of therapy, Pain was reassessed at the end of treatment for each of the three symptoms. Also recurrence, frequency of pain and the use of pain killers were reassessed at end of treatment. The numbers of women at trial entry and at the end of treatment who had either no pain or any pain (mild, moderate or severe) were calculated from the data presented.

The results demonstrated that continues uses of OCPs significantly reduce dysmenorrhea associated with endometriosis compared with the placebo $(2.69 \pm 1.95 \mathrm{p}$. value $<0.001$ for patient group vs. $4.66 \pm 2.17$ p.value 0.022 for placebo group). The recurrence of pain was higher in placebo group than in patient group $(21 \mathrm{vs.} 6$ patients). Also the use of pain killer was higher in placebo group (15 vs. 10 in placebo group and patient group respectively), but there is statistically significant difference between pretreatment and post treatment in both groups.

Dyspareunia score was also reduced after treatment (the pain score reduced to $1.74 \pm 1.65$, p. value $=0.039$, while for placebo group the pain score was $2.29 \pm 2.01, \mathrm{p}$ value $=0.373)$. The recurrence and frequency of pain was low in both groups ( 3 and 6 patient). Also the use of pain killer was low in both groups. There is statistically significant difference between pretreatment and post treatment only in patient group.

Non-menstrual pain was reduced after treatment for patient group $(1.89 \pm 2.05 \mathrm{p}$.value $=0.041)$, while for placebo group there are slight reduction $(2.14 \pm 2.25$, p. value $=0.741)$. The recurrence and frequency of pain was low in both groups ( 5 and 7 patient). Also the use of pain killer was low in both groups. The data shows statistically significant difference between pretreatment and post treatment only in patient group.

Regarding safety, the incidence of irregular uterine bleeding and nausea was higher in the OCP group compared with the placebo group. No serious side effects were noticed during trial.

These results correspond well with the most widely accepted pharmacologic theory that OCs prevent ovulation and suppress the progesterone-driven proliferation of the secretory endometrium during the luteal phase, thereby resulting in a decrease in the volume of menstrual fluid and prostaglandin synthesis and decrease endometriosis associated pain.

In conclusion the present study clearly demonstrated that continues use of low dose OCPs are an effective treatment for pain associated with endometriosis with few adverse effects in women who did not wish to get pregnant in the near future. However longer studies is recommended to evaluate the long term efficacy of oral contraceptive pills in controlling pain of endometriosis as well as the recurrence of pain after discontinuation of therapy.

\section{References}

1. Kodaman $\mathrm{PH}$. Current Strategies for Endometriosis Management. Obstet Gynecol Clin North Am. 2015; 42: 87-101.

2. Al-Jefout M. Brief update on endometriosis treatment. Middle East Fertil Soc J. 2011; 16: 167-174.

3. Harada T, Momoeda M, Taketani Y, Hoshiai H, Terakawa N. Low-dose oral contraceptive pill for dysmenorrhea associated with endometriosis: a placebocontrolled, double-blind, randomized trial. Fertil Steril. 2008; 90: 1583-1588.

4. Harada T, Momoeda M, Terakawa N, Taketani Y. Evaluation of a low-dose oral contraceptive pill for primary dysmenorrhea: a placebo-controlled, double-blind, randomized trial. Fertil Steril. 2011; 95: 1928-1931.

5. Davis LJ, Kennedy SS, Moore J, Prentice A. Modern combined ora contraceptives for pain associated with endometriosis. Cochrane Database Syst Rev. 2009. 